\title{
Entrelacs
}

Cinéma et audiovisuel

$17 \mid 2020$

$N^{\circ} 17$ / Enjeux Audiovisuels du cinéma 360

\section{Un cinéma en devenir :}

le cinéma $360^{\circ}$ en question

\section{Wei-chu Shih}

\section{(2) OpenEdition}

\section{Journals}

Electronic version

URL: http://journals.openedition.org/entrelacs/5937

DOI: 10.4000/entrelacs.5937

ISSN: 2261-5482

Publisher

Éditions Téraèdre

Electronic reference

Wei-chu Shih, « Un cinéma en devenir : », Entrelacs [Online], 17 | 2020, Online since 01 July 2020, connection on 03 July 2020. URL : http://journals.openedition.org/entrelacs/5937 ; DOI : https:// doi.org/10.4000/entrelacs.5937

This text was automatically generated on 3 July 2020 .

Tous droits réservés 


\title{
Un cinéma en devenir :
}

le cinéma $360^{\circ}$ en question

\author{
Wei-chu Shih
}

\section{AU NOM DU CINÉMA}

1 Le 28 décembre 1895 est reconnu comme étant le début de l'histoire du cinéma, ou plus radicalement comme la naissance du septième art, il résulte d'un long processus d'avancées techniques à propos de l'image animée. C'est l'expérience collective qui détermine sa spécificité. Raymond Bellour insiste sur cette expérience qui se définirait par un visionnage collectif d'une durée déterminée dans une salle obscure. Aujourd'hui, les dispositifs de visionnage des films ne se limitent plus à l'écran de cinéma. Non seulement le téléviseur est également devenu un médium de diffusion de film, mais les écrans d'ordinateur, de téléphone portable ou de tablette numérique fournissent des séances ciblant l'individu plus que le collectif grâce au streaming. En l'occurrence, à l'âge numérique, cette diversification des modes de visionnage fait plus écho au Kinétoscope d'Edison qu'au Cinématographe des frères Lumière. Comme le dit Jacques Aumont « le cinéma est un terme fortement polysémique et hétérogène »" la "vraie" histoire du cinéma est "une construction et le produit d'une relation à définir »" Autrement dit, l'histoire du cinéma est variable selon le point de vue adopté. Même si la construction d'une nouvelle histoire n'est pas notre objectif, c'est bien cet esprit relationnel que l'on suit, - la relation entre les dispositifs de l'image animée et ceux de la représentation visuelle -, afin d'élargir la notion de cinéma d'un côté et de faire ressortir les empreintes du cinéma dit conventionnel dans le cinéma 360 taïwanais de l'autre.

2 Les cinéastes taïwanais commencent à tourner des films en réalité virtuelle dès 2017 et il n'est pas surprenant que ce soit Tsai Ming-liang qui ait initié cette expérimentation technologique. Ce cinéaste a assez tôt signalé sa nostalgie de la disparition des salles de cinéma dans Goodbye, Dragon Inn (2003). Il s'éloigne ensuite de plus en plus radicalement des salles au profit de l'espace muséal, théâtral, hôtelier, voire virtuel. Un processus à l'épreuve de la plasticité du film, alors que la salle de cinéma n'est plus l'espace 
privilégié des images cinématographiques chez Tsai. Cette migration spatiale de l'image cinématographique renvoie à la notion d'Expanded cinema - proposé par Gene Youngblood en 1970, le terme marque une transition de l'exposition de l'image mouvante vers l'espace muséal et une corrélation entre le cinéma (à l'égard de son dispositif ou de son contenu au sens large) et l'art contemporain, - réévaluée par Luc Vancheri. Pour lui, le cinéma ne pourrait être « étendu » qu'à condition de considérer :

...le contemporain comme une fonction déterritorialisante du cinéma à partir de laquelle une reconfiguration illimitée des dispositifs et des œuvres est autorisée. Les œuvres cessent ainsi d'être tenues par un même modèle de reproductibilité technique et esthétiques, elles négocient pour elles-mêmes la forme de leur dispositif. $^{3}$

3 Selon Vancheri, le cinéma des premiers temps était en pleine exploration et « s'est tenu à l'écart d'un tel modèle ${ }^{4} »$. Il pense préférable d'interroger les facteurs (techniques ou phénoménaux) qui font varier le cinéma. Autrement dit, si l'on insiste pour conserver le nom de " cinéma ", c'est pour identifier ce qui s'invente sous de nouveau rapports en vue de considérer la manière dont le cinéma fait histoire. C'est la raison pour laquelle le regard de Vancheri se pose sur trois moments où le cinéma s'invente: le moment Lumière, le moment Canudo et le moment Youngblood. Ces trois moments représentent les différentes écritures de l'histoire du cinéma à partir de trois rapports distincts. Respectivement:le cinéma et son invention technologique, artistique puis cybernétique. Un regard archéologique accompagne implicitement l'évolution de la corrélation entre l'homme, la machine et l'image tout au long de son histoire. Il n'est donc plus gênant d'inclure la réalité virtuelle sous la notion " cinéma ». La manière de visionner et de montrer les images mouvantes n'exige plus de représentation authentique comme lors de la fameuse séance des Frères Lumières. Lorsque nous effectuons une réévaluation de l'expérience cinématographique, les questions de la technique du regard et de la culture visuelle sont mises au contact de cette relation triangulaire entre héritages, transformations et transgressions cinématographiques, réinterrogeant ces trois notions dans les œuvres du cinéma $360^{5}$.

4 Le film en réalité virtuelle fournit une vision à 360 pour le spectateur. Alejandro González Iñárritu considère que cette technologie est capable de «briser la dictature du cadre ${ }^{6} »$. Même si son œuvre Carne y Arena est un film VR (selon la catégorisation de Fuchs ${ }^{7}$ ) qui présente une activité sensorimotrice plus active que celle des films dont nous parlerons plus tard, son discours nous permet de réfléchir à la corrélation entre technique du regard et construction du récit à partir du cadre. Est-ce que le cadre, une forme éprouvée pour la focalisation narrative visuelle depuis la Renaissance, a vraiment disparu dans les prémices du cinéma 360 Taïwanais ? Comment la transformation du cadre affecte-t-il la focalisation narrative ? Les cinéastes taïwanais inventent-ils une transformation de cette méthode en s'appuyant sur d'autres formes artistiques?

\section{I - La technique de la focalisation}

5 L'histoire de la technologie de la réalité virtuelle est souvent construite selon une chronologie technique ${ }^{8}$. Or, il est important d'y introduire la focalisation narrative cinématographique ${ }^{9}$, ainsi que les installations immersives parues dans l'histoire du cinéma ${ }^{10}$. Ces perspectives fournissent des discussions intéressantes pour comprendre les caractères esthétiques et les outils propres à ce média. Cependant, elles sont basées 
sur une position relative qui sépare le cinéma de la réalité virtuelle, une opposition entre la notion d'ancien et de nouveau. Il est d'insister sur une évolution visuelle et perceptive (au lieu d'une rupture médiatique) pour répondre aussi bien aux caractères de la réalité virtuelle, - l'immersif, le réalisme et la présence ${ }^{11}$-, qu'à la stratégie esthétique des cinéastes taïwanais.

\section{I-1 - L'évolution du dispositif à vue élargie et du cadre}

6 L'image à 360 donne au spectateur une vision « totale », un sentiment du réel en raison d'une représentation de la proximité géographique ou d'un environnement global où le spectateur a l'impression d'être librement et de découvrir activement. Dans le cinéma «conventionnel », le plan panoramique incarne également ce désir de fournir au spectateur une image quasi-totale. Il traduit une envie, une tendance à élargir l'espace diégétique de l'écran en le prolongeant au hors-champ, dans une durée déterminée. Si la notion totale suppose ici une vue élargie qui tente de donner au spectateur l'impression d'être présent dans l'espace diégétique, la scène de l'image à 360 pourrait trouver son origine dans le "Panorama" inventé par Robert Barker au $18^{\mathrm{e}}$ siècle $^{12}$, époque à laquelle le mot "panorama» est mentionné pour la première fois ${ }^{13}$. Cela signifie une expérience immersive de la perception et une vision élargie. La guerre et le paysage sont souvent les thèmes des peintures de Barker qui installe ses œuvres géantes dans un bâtiment clos en forme d'anneau, où une voûte en verre laisse entrer la lumière afin de donner un effet encore plus naturel et réel. Les visiteurs sont alors englobés par ce dispositif comme s'ils y étaient vraiment présents. Après avoir inventé le Cinématographe, les Frères Lumières créent le «Photorama » en 1900, un appareil capable de capturer et de montrer une vue à 360 . Cette idée est rapidement utilisée pour le cinéma avec le « Cinéorama » créé par Raoul Grimoin-Sanson pour l'Exposition Universelle de Paris en $1900^{14}$. Le Cinéorama est composé de 10 cinématographes disposés en étoile, les images sont projetées sur dix écrans qui entourent les spectateurs en donnant une vue panoramique de Paris. Même si ce gigantesque appareil n’a jamais été exploité, pour Réjane Hamus-Vallée, suite à cette invention, le concept de 360 va connaître de nombreuses variations :

Dès 1955 le procédé Circarama, aussi appelé Circle Vision 360, pour les parcs Disney. La polyvision d'Abel Gance, utilisé pour son Napoléon de 1927, puis les différents écrans larges type CinémaScope qui se multiplient dans les années 1950 sont des versions réduites de ces différents 360 , tout en visant un résultat proche : immerger le spectateur dans le film en lui offrant un champ de vision largement supérieur aux écrans $«$ standards $»^{15}$.

7 En fait, les exemples donnés par Hamus-Vallée ne sont pas strictement des dispositifs d'image à 360 , mais ils offrent une vision plus large qu'un écran standard. Ils cherchent à se rapprocher de la réalité physiologique humaine. Fuchs pose la question de la vision élargie du cinéma 360 alors que les yeux humains, limités à une vision horizontale comprise entre 100 et 210 degrés (en réalité, seulement $110^{\circ}$ ), ne la voient pas: les visiocasques occultent la vision périphérique alors que le mouvement des yeux, généralement de 10 à $20^{\circ}$, est plus faible que celui de la tête ; intentionnellement, l'observateur veut de ne pas voir le « cadre » de l'image (les bords noirs des optiques du visiocasque ${ }^{16}$. Cette limite physiologique et technique du visiocasque implique le même travail essentiel aussi bien pour le cinéaste de cinéma que pour celui du cinéma 360 : la focalisation dans un cadre délimité. En l'occurrence, nous pouvons interroger cette 
domination du «cadre » comme moyen efficace de focalisation narrative, dans le cinéma 360 taïwanais.

8 Le cadre délimite la représentation visuelle du cinéma conventionnel. Selon les recherches de Philippe-Alain Michaud, le concept du cadre pourrait remonter à la Renaissance, grâce à l'invention de la perspective qui crée le proscenium ${ }^{17}$. D'un côté, le cadre résulte d'une transformation géométrique d'un volume sur une surface en créant la profondeur du champ. D'un autre côté, il représente une forme efficace pour attirer l'attention du spectateur afin qu'il se concentre sur la séquence narrative que l'artiste veut exprimer. Dans le contexte de l'histoire du cinéma, ce proscenium qui resserre la concentration visuelle est remplacé par l'écran de cinéma. De plus, la salle obscure du cinéma renforce cette attention et cet effet immersif, englobant le spectateur dans l'environnement diégétique afin qu'il oublie plus facilement le lieu où il se trouve. L'application de ce duo cadre-obscurité ne se limite pas à la salle du théâtre ou de cinéma, mais est également présent dans des espaces d'exposition comme le dit Michaud. ${ }^{18}$ Ainsi, l'anthropologue Franz Boas suggère au musée américain d'histoire nationale de New York de traiter la vitrine comme un écran en proposant un parcours d'exposition à travers un affichage panoramique. Grâce à l'idée de ce duo cadreobscurité, les objets exposés sont placés dans une vitrine éclairée alors que la lumière de la zone d'exposition est atténuée pour pousser les visiteurs à se concentrer davantage sur la scène présentée et à ressentir le message passé par cette installation ${ }^{19}$. En suivant la combinaison de ces deux dispositifs créés dans l'intention d'une expérience immersive, nous comprenons mieux la raison pour laquelle le cadre domine encore dans certaines œuvres du cinéma 360. D'après André Gaudreault ${ }^{20}$, il y a nécessité d'un processus d'institutionnalisation préalable à la naissance d'un nouvel art, il nous est donc permis d'affirmer que le cinéma 360 taïwanais en est à ses "premiers temps ». Nous découvrons qu'une tension persiste encore entre ce nouveau dispositif en phase d'exploration et ses prédécesseurs. Surtout, le concept du cadreécran est révélateur de cette tension.

\section{I-2 - Le concept du cadre-écran : l'agent de la focalisation}

\section{I-2-A - LE CONCEPT DU CADRE-ÉCRAN}

9 Le film Your Spiritual Temple Sucks de John Hsu, l'un des cinéastes taïwanais pionnier du cinéma 360 en 2017, est inspiré du rituel taoïste «les yeux bandés» de la croyance populaire taiwanaise ${ }^{21}$. Le cinéaste cherche déjà ici à concentrer l'attention du spectateur par le cadre en divisant verticalement l'espace diégétique en deux hémisphères, l'un montrant la vie réelle du personnage et l'autre son Temple Spirituel ( 元神宮). Le dieu du tonnerre, le gardien de l'histoire, donnera aux spectateurs des indices lorsqu'ils seront censés regarder l'hémisphère avant ou arrière. Cette œuvre propose des scènes en "pixelart» et des touches humoristiques lors de la visite du domaine spirituel, mais l'idée du cadre-écran est encore limitée par nos habitudes visuelles qui consistent à se déplacer dans les images de manière conventionnelle.

De plus, cette disposition basée sur le cadre de l'écran rappelle au spectateur son statut de public. Dans ce dispositif, son point de vue est distant. En d'autres termes, le récit n'est pas construit autour d'un personnage incarné par le spectateur. Ne pouvant interagir, il ne peut pas non plus dérouter volontairement le développement de l'histoire. Les consignes narratives l'obligent à suivre l'histoire attentivement, alors que 
le spectateur découvre l'espace diégétique plutôt visuellement et non physiquement. Ici, le spectateur observe les actions en sachant qu'il est mis à la troisième personne, une position distante et passive. Ainsi, le quatrième mur du théâtre ou le cadre de l'écran se transforme en un film transparent invisible pour écarter la scène du lieu où se situe le spectateur.

Dans Le Train Hamasen de Kuan-yuan Lai, le cadre de l'écran est associé aux origines du cinéma. Dans une scène, le spectateur suit la dame du chariot de collation dans la salle de cinéma. Lorsqu'elle tourne à gauche en passant devant l'écran, le corps du spectateur traverse l'écran jusque dans un autre monde où il se retrouve à regarder l'arrivée d'un train avec une foule de gens. L'image de la dame au chariot de collation, encadrée par un écran de cinéma, est ensuite projetée derrière le spectateur. Il s'agit d'une scène qui montre non seulement la forte ambition du cinéaste de progresser dans un champ de vision à 360 , mais qui fait également allusion à L'Arrivée du train en gare de la Ciotat qui a profondément changé la perception visuelle de l'homme. Ainsi, Lai indique que l'histoire de la représentation visuelle est sur le point d'entrer dans une nouvelle ère technologique.

\section{I-2-B - L'ÉCRAN COMME LE MIROIR}

Dans Afterimage for Tomorrow, Singing Chen crée une scène divisée par un rayon de lumière en deux hémisphères avec un groupe de danseurs restant dans l'une de ces hémisphères et leurs reflets dans l'autre. Alors que ces danseurs hypnotisés somnolent, leurs réflexions continuent de se tortiller en portant leurs visiocasques de VR. Positionné au centre de la sphère, le spectateur peut observer librement les deux côtés. À travers cette mise en abyme du cinéma 360 , la cinéaste réfléchit à la corporéité dont le spectateur ne peut pas s'échapper.

13 Au cinéma, l'effet miroir s'associe de plusieurs manières à la question de la " réflexivité ». Il est basé sur le concept d'imaginer l'écran / le cadre comme un miroir dans lequel « le moi spectatoriel » signifie la subjectivité du spectateur représentée par le point de vue à la première personne sur l'écran. Autrement dit, la « mise en abyme » pourrait aussi figurer une autre forme de réflexivité. Dans le cinéma 360, ni écran, ni cadre n'existent physiquement dans le champ de vision à 360 degrés. Or, Chen transforme l'effet miroir en deux hémisphères qui permettent au spectateur de comprendre comment il pourrait apparaitre et se comporter avec un visiocasque. Les corps des danseurs endormis font écho au corps dans le coma, état que le son d'un appareil respiratoire suggère dès le début du film. La disposition de cette scène cherche à intégrer dans son expérience de visiocasque une image obscure de la mort. Cette scène prouve non seulement que la technologie de la réalité virtuelle est capable de créer une perception transcendante, mais confirme également que notre corps n'a nulle part où s'échapper. Dans les films, l'effet miroir est créé en fonction d'une disposition dichotomique de l'espace entre l'écran et la salle de cinéma. Dans Afterimage for Tomorrow, cet effet est incarné par la disposition de couches en forme de cercles concentriques au centre desquels le spectateur est placé, en s'appuyant sur la relation entre le regard du spectateur, l'objet à mettre en miroir et l'image réfléchie de l'autre côté du miroir. Cet effet n'agit plus sur l'itinéraire de la réflexion mais sur la réfraction à partir de laquelle le corps du spectateur complexifie la notion du regard, autant au sens narratif que du point de vue de la perception. 
Your Spiritual Temple Sucks reprend le concept du cadre de l'écran pour concentrer le regard du spectateur, alors que l'écran est l'emblème d'une vieille technique visuelle de la perception à dépasser dans Le Train Hamasen. Dans Afterimage for Tomorrow, l'effet miroir est interprété poétiquement pour une allusion critique de la mortalité corporelle. Que ce soit par la construction de l'espace diégétique ou par la transformation de l'idéologie du regard à partir du cadre, la conversion du "regard " dans le cinéma 360 met en lumière la réflexion d'une technique de celui-ci où le corps du spectateur se problématise. En d'autres termes, «le regard » n'est plus simplement visuel mais corporel, renforcé par la nature du média issue de la superposition entre la caméra et le spectateur. Le regard se modifie en conséquence comme l'agent narratif et perceptif au niveau de la focalisation narrative. C'est la raison pour laquelle l'appellation "spect-acteur" reprise par Fuchs ${ }^{22}$ répond mieux à l'ambivalence de l'observateur du cinéma 360.

\section{Le corps-fantôme : l'ambivalence entre ici et ailleurs}

Le corps du spectateur évolue - ou est transformé - entre le corps écarté du quatrième mur du théâtre et le corps-miroir de l'écran de cinéma. Différentes installations se créent à partir du concept de cadre et de la logique narratologique que le corps représente. André Bazin a mentionné la distinction proposée par Rosenkrantz entre le spectateur de théâtre et celui de cinéma. Le premier est forcé à un engagement actif pour s'identifier à la scène théâtrale alors que l'identification à l'espace diégétique est plus naturelle pour le deuxième ${ }^{23}$. Ce processus naturel est interprété par Christian Metz comme le stade du miroir lacanien, et est comparé au rêve par Jean Mitry via la psychanalyse. Cependant, comme nous l'avons mentionné plus tôt, qu'il s'agisse de théâtre, de cinéma ou d'installation dans un musée, la frontière de l'image persiste. C'est l'obscurité qui, en dirigeant le regard, donne l'illusion de la disparition de cette frontière pour que le spectateur soit "présent dans l'image ». Or, le spectateur est immédiatement "présent dans l'image " quand il porte le visiocasque. En raison du « superpositionnement » entre la caméra et le spectateur, ce dernier est placé au centre de l'environnement en réalité virtuelle. Cette technologie isole radicalement le spectateur et l'installe dans un autre univers spatiotemporel. Pour Benjamin Hoguet, "la présence [du spectateur] est donc le moteur de la réalité virtuelle. Il nous permet de nous imaginer autre et ailleurs à la fois ${ }^{24}$. » Le visiocasque fournit l'effet de la réalité virtuelle à travers une double isolation: l'isolation physique par le casque; l'isolation psychique par le corps fantôme, autrement dit un corps virtuel sera créé pour expérimenter l'environnement virtuel. De ce fait, cette expérience évoque l'ambivalence de la perception corporelle. Char Davies parle d' " un mode de perception inhabituel» et d'«un sentiment paradoxal d'être en et hors du corps $»^{25}$. Cette ambivalence vient du fait que deux actions, témoigner et jouer, figurent autant l'une que l'autre le statut qu'assume le spectateur par l'usage du visiocasque. Fuchs propose le mot «spect-actor" pour souligner, à travers ces deux actions, l'activité sensorimotrice active dans le film VR par rapport au Vidéo $360^{26}$. L'emprunt narratologique de la perspective à la première et troisième personne est rapidement pris en compte par John Mateer ${ }^{27}$. Le premier incarne l'un des rôles principaux qui affecte le développement de l'intrigue ; le deuxième est un témoin qui observe ce qui se passe autour de lui. Or, cette catégorisation est à la base de la narratologie, elle n'arrive cependant pas à mettre en lumière un statut trinitaire regard/rôle/perception 
incarnée du spectateur. Il est aussi pertinent de considérer la présence du spectateur du cinéma 360, qui est souvent intégrée dans la construction du récit, l'histoire se développant autour de ce regard présent, voire dominant. Autrement dit, le regard du spectateur est devenu à la fois une incarnation de la vision (au niveau narratologique) et une perception incarnée (au niveau cinétique). Comme nous en avons parlé cidessus, le cadre/écran en tant qu'idée a profondément influencé, entre autres, les trois films mentionnés. Nous allons maintenant observer comment le cadre/écran est métamorphosé en moteur perceptif d'une expérience cinétique.

\section{II-1-L'incarnation d'un regard virtuel}

16 Selon Gilles Deleuze la notion de virtuel qui ne s'oppose pas au réel, mais à l'actuel ${ }^{28}$. Pierre Lévy souligne également que le virtuel existe « en puissance et non en acte [,] (...) tend à s'actualiser, sans être passé cependant à la concrétisation effective ou formelle ${ }^{29}$. » Pour revenir à notre argumentation selon laquelle le cadre/écran persiste, celui-ci n'est pas physiquement concrétisé, mais transformé en agent d'intrigue. Cet agent est immatériel, comme une force qui sera identifiée par l'expérience de voir et de sentir cinétiquement l'histoire. Si nous parlons du statut trinitaire du spectateur, ou de l'ambivalence de son corps, c'est parce qu'un regard virtuel coexiste avec le point de vue subjectif lorsque le regard subjectif perd sa signification particulière, et ce à cause de la superposition de la caméra et du spectateur. Ce regard virtuel résulte d'une énergie qui pourrait être identifiée par «l'expérience encadrée ».

"L'expérience encadrée » fait référence à l'expérience créée par l'écran comme substrat. Dans les films, le cadre/écran détermine l'espace dans lequel le public perçoit les images en mouvement. Un tel cadre/écran n'existe plus dans les œuvres en réalité virtuelle, mais nous considérons «le cadre» de manière abstraite, "perspective encadrée» et "perspective expérimentée », pour identifier la corporalité formée respectivement dans la diégèse à la surface du cadre et au sein du cadre.

La "perspective encadrée " désigne souvent le point de vue à la première personne. Lorsque le point de vue subjectif du spectateur est dirigé vers un personnage spécifique, il trouvera sa vision plus ou moins limitée, car il doit voir à travers les yeux de ce personnage. Ainsi, contrairement au point de vue à la troisième personne, une position distanciée, le spectateur est invité à " contempler » l'articulation des actions et des péripéties de l'histoire. Le verbe « contempler » signifie ici non seulement regarder, mais bien réfléchir par soi-même; en observant une scène, le spectateur est bien conscient du rôle qu'il joue et de son statut dans ce scénario spécifique. "On ne voit qu'à travers le rôle assigné » signifie que le spectateur est limité à une condition visuelle prédéfinie, empruntée à la focalisation interne de la narratologie

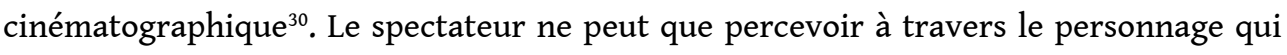
lui est assigné. Malgré un environnement à 360 , l'énergie du cadre amorce une vision encadrée non seulement visuelle mais aussi par la perception incarnée au sens cinétique. Pour cette raison, la perspective encadrée est liée à une expérience de cadrage, une corporalisation du cadre. C'est le cas dans Mr. Buddha (2018) de Lee Chung. Le spectateur se trouve à l'intérieur d'une ancienne tête de Bouddha qui doit être volée par une bande de cambrioleurs. Le spectateur est coincé dans un corps immobile et suit une aventure par les déplacements et les manipulations de cette bande. 
19 Pourtant, la perspective se distingue légèrement de la focalisation interne cinématographique, car le spectateur perçoit le mouvement dans l'image. Même si l'interactivité entre le spectateur et l'environnement en réalité virtuelle est censée définir la nature de la réalité virtuelle dans l'objectif de simuler une sensorimotricité, l'orientation spatiale provoque assez souvent des sensations d'inconfort (vertige, nausée...), liées au système physiologique du conduit auditif humain, quand le mouvement devient brutal. La caméra reste le plus souvent immobile pour capturer une scène et chaque mouvement doit être bien contrôlé et réfléchi. Le travelling est souvent reproduit dans le cinéma 360 , alors qu'il pourrait être réalisé de manières diverses à l'aide d'un drone ou d'effets spéciaux. Le cinéaste Lee décide de placer le spectateur/la tête de Buddha dans un camion qui produira un travelling. Comme mentionné plus tôt, la perspective encadrée domine la manière de découvrir et d'expérimenter l'histoire. Dans la scène où la tête de Buddha est déposée dans le compartiment arrière du véhicule, le hayon est ouvert comme une "fenêtre » offerte au spectateur pour qu'il ait une vision de l'extérieur du camion. Le personnage A-Che, un cambrioleur débutant, tue son boss Dong-Tzu puis occupe le siège conducteur et s'enfuit. À partir de ce moment, un champ de vision "supplémentaire » est fourni par cette mise en scène/espace, le spectateur perçoit un mouvement de fuite en voyant l'éloignement du corps laissé par terre jusqu'à sa disparition du cadre primordial ${ }^{31}$. Cette reproduction $\mathrm{du}$ travelling rend accessible une perception physique $\mathrm{du}$ mouvement et psychologique du personnage avec qui une position de hors-jeu est effectuée. Autrement dit, la perspective subjective du film se déplace entre celle à la première personne (de l'autre) et celle à la troisième. Si le spectateur se superpose à un objet, cela suppose une position témoin qui observe à distance les actions qui l'entourent. Or, le travelling permet au spectateur de glisser vers l'émotion impétueuse d'A-Che, le juxtaposant à la tension psychologique de la scène. "La perspective expérimentée " relève ainsi une double qualité de la notion de mouvement: le mouvement physique (par la forme ou la vitesse de l'image-mouvement) et le mouvement psychologique du temps subjectif (du personnage ou de la scène).

L'incarnation d'un regard virtuel met en accent les déplacements constants entre l'extériorité et l'intériorité du cadre de l'écran, entre les perspectives narratologiques et les mouvements cinématographiques.

\section{II-2 - La théâtralité du corps bloqué}

\section{II-2-a - La technique de la focalisation dans la scène à 360}

21 Pour Singing Chen, lorsque la caméra se situe au centre d'un environnement en réalité virtuelle, l'enchaînement des actions ou des actes dans cette vision " totale " s'adapte mieux à la mise en scène théâtrale. Selon elle, le film en VR est plus théâtral que cinématographique ${ }^{32}$. C'est que nous pensons l'enchaînement des scènes plus que celui des plans pour dérouler l'histoire. Ainsi, Jessica Brillhart, la fondatrice de Vrai Picture, ancienne employée de l'entreprise Google, propose "Probabilistic Experiential Editing ${ }^{33}$ " comme méthode de focalisation narrative dans les œuvres en VR. En raison de la présence du spectateur, la focalisation narrative est étroitement liée à l'expérience spectatorielle. Il est donc nécessaire de définir d'abord le " point d'intérêt » (POI, point of interest) du spectateur. Par exemple, dans une image qu'elle appelle " Hero's Journey ", le spectateur se situe au centre d'un cercle concentrique, tandis que les points blancs 
sont le $P O I$ au début d'une scène, et les points noirs sont le POI à la fin de cette scène. Afin de diriger le spectateur vers le POI à la fin de la scène, la musique, les effets sonores, l'action théâtrale, la couleur peuvent être utilisés pour guider le public vers le «point de sortie ». Dans le montage du film, le point d'entrée (in-point) et le point de sortie (out-point) sont le début et la fin d'un plan, tandis qu'en VR, le point d'entrée est le début de l'expérience du spectateur et le point de sortie en est la fin. Par conséquent, grâce au POI initial défini dans chaque zone du cercle, le spectateur suit le chemin prédéfini par le créateur du cinéma 360.

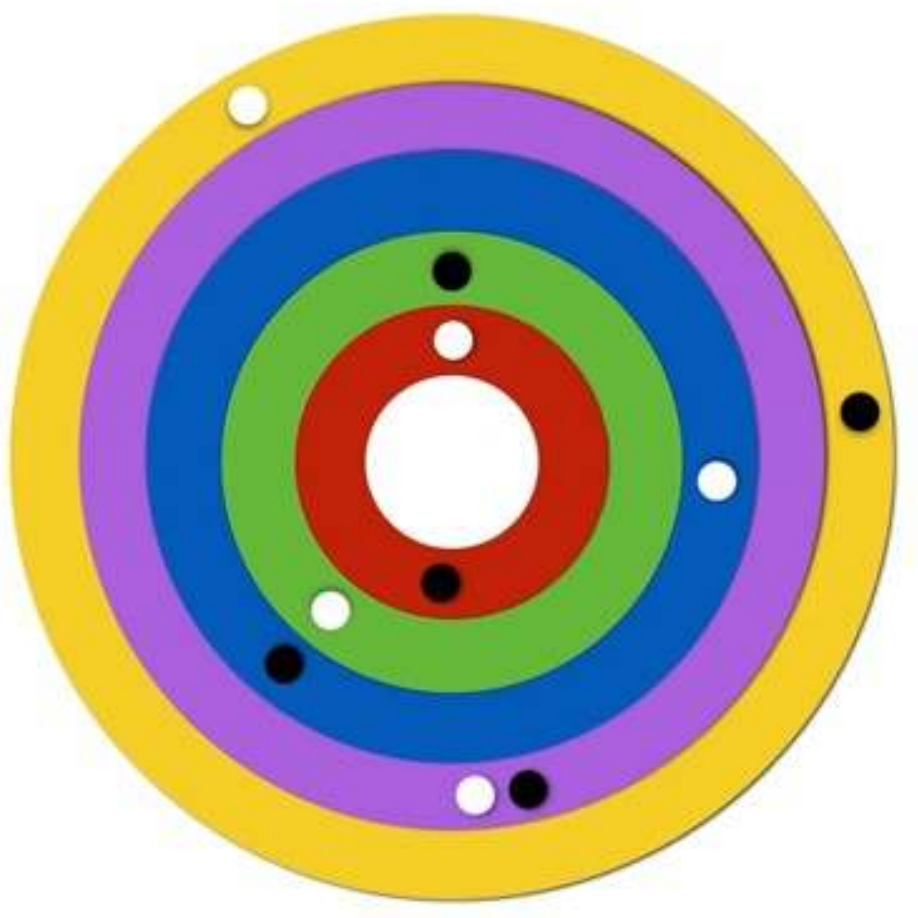

Figure 1: The Hero's Journey de Jessica Brillhart

Chen utilise la lumière (chandelles ou éclairage) pour marquer le point d'entrée, alors que l'obscurité (la disparition des sources lumineuses) est le point de sortie pour passer à la scène suivante ou le point de transition pour dérouler l'action. Les points d'entrée et de sortie se trouvent dans la même scène capturée par un plan fixe, l'idée du planséquence, méthode cinématographique héritée du septième art, est en l'occurrence appropriée. Mr. Buddha est réalisé en un "faux » plan-séquence avec mouvements de caméra, c'est-à-dire que des fondus au noir servent pour les raccords qui correspondent parfaitement au tournant de l'intrigue. Cela donne l'impression du planséquence ininterrompu.

\section{II-2-b - La théâtralité du plan-séquence : le trompe-le temps}

La plupart des plans-séquence sont réalisés par un plan fixe dans le cinéma 360, tandis que le jeu des comédiens doit être effectué en une seule prise. De ce fait, nous considérons l'expressivité corporelle des acteurs comme l'appui du déroulement de l'histoire du cinéma. André Bazin distingue le théâtre du cinéma au niveau de la mise en " présence » des acteurs, «[le cinéma] le fait à la manière d'un miroir ${ }^{34}$ ». La notion de théâtralité s'associe étroitement à la présence des acteurs. Jacques Araszkiwiez 
relève les différences de cette notion chez Bazin et Barthes: «pour Bazin, c'est la présence elle-même de l'acteur qui induit la théâtralité; pour Barthes, c'est le sentiment de cette présence qui provoque la même induction ${ }^{35}$. "

En poursuivant ces réflexions, le cinéma 360 semble brouiller la frontière entre le cinéma et le théâtre, sa qualité esthétique trouve à la convergence de ces deux arts. Nous avons mentionné que Singing Chen utilise la lumière et l'obscurité comme points d'entrée et de sortie au niveau de la focalisation d'une scène. Dans une scène où un danseur s'approche progressivement du spectateur, le danseur apparaît chaque fois de plus en plus proche, sous un faisceau de lumière différent, sans que l'itinéraire soit enregistré. Le corps du danseur signale les temps disparus. L'obscurité soutient la réalisation d'un "trompe-le temps » dans le plan séquence grâce à la malléabilité de l'image numérique. La plasticité temporelle est désormais réalisable dans un planséquence.

Lorsque le plan-séquence coïncide à l'expressivité corporelle des acteurs, le fait est que "la présence " ne désigne pas seulement celle du spectateur, mais aussi celle des acteurs. La mise en «relief » de leur présence en image 3D renforce une narrativité de l'interactivité sensorimotrice, alors que le regard du spectateur déclenche une tension entre un corps dynamique et un corps observateur, qui marque l'ambivalence de son corps en répondant au rapport entre théâtre et cinéma 360.

\section{II-2-c - La théâtralité du plan-séquence : le corps-fantôme}

Dans Home (2019) de Kidding Hsu, le cinéaste place le spectateur dans un fauteuil roulant, lui assignant un rôle d'A-ma (grande mère en taïwanais) dans un état végétatif. Ce film est réalisé en un plan-séquence qui rappelle Hou Hsiao-hsien, chez qui le planséquence est réalisé pour capturer le moindre détail de la vie quotidienne en en montrant la temporalité continue, souvent lors d'une scène de repas de famille. Home raconte un après-midi de réunion familiale dans une maison de style japonais lors de la fête des bateaux-dragons. Tous les membres de la famille s'approchent sans relâche de A-ma en la saluant, lui parlent même si elle n'est pas capable de répondre ou de réagir sur aucun sujet. Ils la quittent après avoir pris une photo de famille et le film se termine sur la scène où la soignante immigrée laisse $\mathrm{A}$-ma devant la télévision et se penche sur son téléphone portable dans son coin. La mise en scène théâtrale permet au spectateur d'observer les détails décoratifs, les moindres actions prenant place dans les différentes zones de la scène. Surtout, le positionnement du spectateur à la place d'A-ma révèle brillamment la réalité cruciale contemporaine de la situation des vieillards. La réunion familiale imite la scène théâtrale: tous les personnages saluent un à un A-ma/le spectateur. Les gestes des acteurs relativisent la place du spectateur dans le dispositif. Il est un personnage ou un simple témoin dont le corps virtuel provient du corps physique du spectateur. L'interactivité est cependant interprétée différemment par un corps dédoublé en corps d'observateur et en corps de perception. Le corps d'observateur est libre de voir sans contrainte narratologique, alors que le corps de perception est censé prendre le rôle qu'on lui assigne dans un espace diégétique où il ressent et expérimente.

Le corps de perception est aussi un corps virtuel. Cette virtualisation du corps réinvente l'expérience de voir par le corps. Pour Lévy, la virtualisation du corps est « une réinvention, une réincarnation, une multiplication, une vectorisation, une hétérogenèse de l'humain ${ }^{36}$.» Le cinéma 360 engendre un corps-fantôme à prendre 
comme une prothèse du spectateur pour "voir/sentir plus ». S'il est un faux VR pour certains spécialistes de cette technologie en raison du manque d'interactivité, c'est exactement par cette restriction de la mobilité chez le spectateur qu'une intensité pourrait être parfaitement interprétée comme une frustration. En prenant le cinéma 360 comme la répression d'un corps libre, une stratégie à la fois esthétique et critique est engagée par les deux cinéastes Hsu et Chen. Tsai Ming-liang ne s'inscrit pas dans la lignée de cet esprit critique, il se questionne principalement sur ce que pourrait encore être le cinéma ${ }^{37}$. Cependant, il partage inconsciemment l'idée d'une corporéité virtuelle à partir de l'acte de voir dans un but de plus éprouver le temps.

Depuis ses derniers films, Tsai ne s'intéresse plus à l'histoire mais à l'acte de voir. Par la longueur de ses plans fixes dans lesquels il ne se passe souvent rien, le cinéaste invite le spectateur à contempler les personnages, l'espace et le temps. Au lieu de penser la nouveauté de cette technologie ou sa possibilité, le cinéaste reprend la même philosophie en construisant des scènes de longue durée pour voir plus et plus attentivement. Son film de 56 minutes se compose d'une dizaine de scènes, le spectateur y observe, entre autres, ses acteurs fétiches Xiao Kang, Lu Yi-ching et Chen Shiang-chyi. Cette position témoin, perspective à la troisième personne de l'espace diégétique, est vraisemblablement un regard fantomatique, dérangeant et incertain. Nous voyons que Xiao Kang est dans un canapé, un patch électronique dans le cou, Shiang-chyi reste immobile sur une cuvette, mais ils s'enferment dans leurs propres états d'esprit qui réduisent davantage l'interactivité potentielle offerte par le cinéma 360. De plus, cette présence fantomatique ne désigne pas non plus une volonté de se déplacer librement. La longue durée du plan fixe provoque une envie d'échappement chez le spectateur. L'impression d'un environnement clos est évoquée par une double isolation : l'ignorance des personnages et la ruine partout. Cela renvoie parfaitement à la proposition issue de son titre originel 《家在蘭若寺》 (Jia zai lanresi, la ville natale au temple orchidée). Le temple orchidée est une cité de fantômes apparue dans un recueil de contes en chinois classique Contes extraordinaires du pavillon du loisir, paru en 1766. L'intention du cinéaste, est de simuler la ruine d'un théâtre fantomatique qu'il nous invite à contempler. Le sentiment d'isolation provient de la séparation de deux mondes hétérogènes, l'acte de voir n'est pas simplement plaisir de la découverte, mais passe également par la perception, l'angoisse de cet enfermement spatio-temporel. L'acte de voir est dirigé vers une contemplation forcée par une durée excessive du plan fixe, pour témoigner d'une corporéité de la perception temporelle, un corps-fantôme est ainsi engendré en devenant le lieu de l'empreinte du temps, figuré par une opération réflexive entre les espaces-temps hétérogènes.

\section{Conclusion}

Leon Battista Alberti considère, dans son livre De Pictura paru en 1435, le cadre rectangulaire du tableau comme une fenêtre qui ouvre en racontant une histoire pour le public. En accompagnant un développement technologique en vue d'explorer les expériences variées de la culture visuelle, cette tradition de la représentation visuelle dans un cadre persiste jusqu'à aujourd'hui. L'engouement pour la réalité virtuelle qui fait disparaître cette contrainte du cadre de la réalité virtuelle est compréhensible. Nous considérons le cadre dans le cinéma 360 comme une continuation de son évolution historique. Les anciens dispositifs visuels ont préparé le développement 
d'une culture visuelle à long terme. Il n'y a pas de rupture de la culture visuelle, mais une évolution. De plus, puisque le cinéma 360 est encore en phase d'exploration et que sa spécificité reste à définir, l'enquête sur les emprunts artistiques à d'autres formes d'art est importante.

Une analyse à partir de la technique de la focalisation au sens narratologique et l'examen de la théâtralité aident à comprendre l'ambivalence du corps du spectateur engendrée par ses déplacements entre "ici » et «ailleurs". L'image à 360 crée une expérience de juxtaposition et de conversion entre différentes identités et perspectives, et la « présence » qui signifie à la fois « ici » et « ailleurs » renforce la complexité de ce mode de perception.

La réflexion articulée entre le concept du cadre, la migration des formes artistiques et l'ambivalence du corps du spectateur mettent en évidence la multiplication du corpsfantôme. Le corps-fantôme se définit comme une prothèse permettant de percevoir l'atmosphère de la scène et de ressentir les émotions des personnages. Le cinéma 360 offre en permanence des va-et-vient entre vécu et transcendance.

\section{BIBLIOGRAPHY}

\section{Bibliographie}

Araszkiwiez, Jacques, «La Génèse de la théâtralité » dans Cinéma et théâtralité, sous la direction de Christine Hamon-Sirejols, Jacques Gerstenkorn, André Gardies, Lyon : Aléas, 1994.

Aumont Jacques, «L'Histoire du cinéma n'existe pas », dans CinémaS, vol. 21 N²-3 printemps, 2011.

Bazin, André, Qu'est-ce que le cinéma ?, Paris : Cerf, 1976.

Comment, Bernard, Le XIXe siècle des panoramas. Paris : Adam Biro, 1993.

Char, Davies. «Changing Space: Virtual Reality as an Arena of Embodied Being» dans. The Virtual Dimension: Architecture, Representation, and Crash Culture, ed. par John Beckman, New-York : Princeton Architectural Press, 1998.

Dooley, Kath, « Storytelling with virtual reality in 360-degrees: a new screen. grammar », Studies in Australasian cinema, Vol $11 \mathrm{~N}^{\circ} 3,2017$.

Fuchs, Philippe, Théorie de la réalité virtuelle - Les véritables usages, Paris : Presses des Mines, 2018.

Fuchs, Philippe (dir.), Le Traité de la réalité virtuelle - volume 1 : fondements et interfaces comportementales, Paris : Presses des Mines, 2003.

Florentina, Nicolae Dana, «Spectator Perspectives in Virtual Reality Cinematography. The Withness, the hero and the Impersonator »: http://ekphrasisjournal.ro/docs/R1/20E10.pdf, 20 novembre 2018.

Gauldreault, André, Cinéma et attraction - pour une nouvelle histoire du cinématographique, Paris : CNRS, 2008.

Gaudreault, André et Jost, François, Le Récit cinématographique, Paris : Armand Colin, 2005. 
Hamus-Vallée, Réjane, «Le Documentaire en 360, un point de vue impossible ? Étude de NYT VR et Arte $360 »$ dans Studies in French Cinema, Vol. 18, NO., 3 février 2018.

Hoguet, Benjamin, La Grammaire de la réalité virtuelle, Paris : Dixit, 2017.

Pierre, Lévy, Qu'est-ce que le virtuel ?, Paris : La Découverte, 2019.

Mateer, John, «Direction for Cinematic Virtual Reality: how the traditional film. director's craft applies to immersive environments and notion of presence », Journal of Media Practice, Vol 18, N¹, mai 2017.

Meusy, Jean-Jacques, «L'Énigme du cinéorama de l'Exposition Universelle de 1900 », Archives 37, 1991.

Michaux, Emmanuelle, Du panorama pictural au cinéma circulaire, Paris : L'Harmattan, 1999.

Michaud, Philippe-Alain, Sur le film, Paris : Macula, 2016.

Michaud, Philippe-Alain, Le Mouvement des images, Paris : Centre Georges Pompidou. Service Commercial, 2006.

Vancheri, Luc, Le Cinéma ou le dernier des arts, Rennes : PUR, 2018.

\section{NOTES}

1. Jacques Aumont, "L'Histoire du cinéma n'existe pas », dans CinémaS, vol. $21 \mathrm{~N}^{\circ} 2-3$ printemps, 2011, p. 155.

2. Ibid., p. 163.

3. Luc Vancheri, Le Cinéma ou le dernier des arts, Rennes : PUR, 2018, p. 289-290.

4. Ibid., p. 29.

5. Philippe Fuchs fait une distinction technique entre « vidéo 360 » et «film VR » dûe à l'absence ou à la présence d'activités sensorimotrices, réalisées par l'usager. Pour la vidéo 360, la liberté de déplacement est restreinte, l'usager ne peut que tourner la tête pour observer les objets, les personnages et la scène. En revanche, il peut (inter)agir volontairement dans l'environnement de la réalité virtuelle. Selon Fuchs, il n'est pas un observateur passif, comme celui de la vidéo 360, mais il est « actif physiquement ». Philippe Fuchs, Théorie de la réalité virtuelle - Les véritables usages Paris: Presses des Mines, 2018, p. 323. Nous prenons le terme « cinéma 360 » appartenant à la catégorie « vidéo 360 » de Fuchs, le mot « cinéma » est supposé ici comme un critère esthétique qui problématise la mise en rapport avec cette nouvelle technologie. Le terme "observateur passif» est relatif à l'usager du film VR. Ce dernier a plus de liberté pour interagir dans l'environnement de la réalité virtuelle. Nous ne développons pas ces deux termes ici, mais introduisons la sensorimotrice au niveau technologique proposé par Fuchs.

6. La phrase originale est «Break the dictatorship of frame ». Voir la communication de presse de Carne $y$ Arena: https://carneyarenadc.com/ Carne\%20y\%20Arena\%20DC\%20Press\%20Release\%203\%2026\%202018.pdf, consultation le 30/03/2019.

7. Philippe Fuchs, op. cit., p. 328-329.

8. «Le Sensorama» de Morton Heilig en 1957 est souvent cité comme point de départ de l'histoire de la réalité virtuelle et le dispositif de "The Sword of Damocles " inventé par Ivan Sutherland en est la seconde étape. Ensuite, on introduit Thomas Furness, créateur de la série « Visually Coupled Airborne Systems Simulator", par exemple The Super Cockpit, qui aidait à l'entraînement de la navigation aérienne pour les pilotes américains dans les années 80 . Bastien $\mathrm{L}$, "L'Histoire de la VR en 7 étapes: de la science-fiction à votre salon» dans Le Magazine de la réalité virtuelle \& augmentée, https://www.realite-virtuelle.com/histoire-vr-7-etapes-1511/, 15 novembre 2017, consultation le 30 janvier 2020. 
9. Kath Dooley, "Storytelling with virtual reality in 360-degrees: a new screen grammar ", Studies in Australasian cinema, Vol $11 \mathrm{~N}^{\circ} 3,2017$, p. 161-171. John Mateer, «Direction for Cinematic Virtual Reality: how the traditional film director's craft applies to immersive environments and notion of presence » dans Journal of Media Practice, Vol 18, N 1, May 2017, p. 14-25. Dana Florentina Nicolae, « Spectator Perspectives in Virtual Reality Cinematography. The Withness, the hero and the Impersonator » : http://ekphrasisjournal.ro/docs/R1/20E10.pdf, le 20 novembre 2018.

10. Réjane Hamus-Vallée, «Le Documentaire en 360, un point de vue impossible ? Étude de NYT VR et Arte $360 »$ dans Studies in French Cinema, Vol. 18, NO., le 3 février 2018, p. 223-238.

11. Philippe Fuchs (dir.), Le Traité de la réalité virtuelle - volume 1 : fondements et interfaces comportementales Paris: Presses des Mines, 2003. p86.

12. Bernard Comment, Le XIXe siècle des panoramas. Paris : Adam Biro, 1993, p. 13-14.

13. Emmanuelle Michaux, Du panorama pictural au cinéma circulaire, Paris : L'Harmattan, 1999, p. 15.

14. Jean-Jacques Meusy, «L'Énigme du cinéorama de l'Exposition Universelle de 1900 » dans Archives 37, 1991, p. 1-16.

15. Réjane Hamus-Vallée, op. cit., p. 224.

16. Philippe Fuchs, Théorie de la réalité virtuelle - Les véritables usages, op. cit., p. 324.

17. Philippe-Alain Michaud, Sur le film, Paris : Macula, 2016, p. 14-20.

18. Philippe-Alain Michaud, Le Mouvement des images, Paris : Centre Georges Pompidou Service. Commercial, 2006, p. 16-17.

19. Ibid., p. 19.

20. André Gauldreault, Cinéma et attraction - pour une nouvelle histoire du cinématographique, Paris : CNRS, 2008, p. 119.

21. "Les yeux bandés » (雚落陰) est une pratique rituelle qui permet la communication entre notre monde et celui des défunts. La séance est animée par un chaman dont le corps devient un médium transmettant les messages du disparu au vivant aux yeux bandés de rouge.

22. Selon Fuchs, le statut du "spect-acteur» chez l'usager de visiocasque VR, parce qu'il "pourrait manipuler des objets, se déplacer librement dans la scène, intervenir dans le déroulement du scénario ou communiquer avec d'autre personne, immergées simultanément, ou avec des personnages virtuels. Philippe Fuchs, Théorie de la réalité virtuelle, op., cit., p328.

23. André Bazin, Qu'est-ce que le cinéma ?, Paris : Cerf, 1976, p. 153.

24. Benjamin Hoguet, La Grammaire de la réalité virtuelle, Paris : Dixit, 2017, p. 21.

25. Les phrases originales sont " unusual modes of perception » et " paradoxical sense of being in and out of the body ". Pour voir son idée complète : Char Davies. "Changing Space: Virtual Reality as an Arena of Embodied Being » dans The Virtual Dimension: Architecture, Representation, and Crash Culture, ed. par John Beckman, New-York : Princeton Architectural Press, 1998. p144-155.

26. Philippe Fuchs, Théorie de la réalité virtuelle - Les véritables usages, op. cit., p. 323.

27. John Mateer, op. cit., p. 14-25.

28. Gilles Deleuze, Différence et répétition, Paris : PUF, 1993, p. 273.

29. Pierre Lévy, Qu'est-ce que le virtuel, Paris : La Découverte, 2019, p. 13.

30. André Gaudreault et François Jost, Le Récit cinématographique, Paris : Armand Colin, 2005, p. 138.

31. Selon Benjamin Hoguet, un « cadre primordial » désigne $100^{\circ}$ de la sphère face au spectateur et un « hors-champ primordial » suppose le reste de la sphère de l'espace diégétique de la réalité virtuelle. Benjamin Hoguet, op. cit., p. 67.

32. Dans une interview avec Singing Chen datée du 25 février 2019, Chen indique qu'elle réalise un travail plus théâtral que cinématographique pendant le tournage d'Afterimage for Tomorrow.

33. Jessica Brillhart, "In the Blink of a Mind-Prologue » et «In the Blink of a Mind-Attention », sur la plateforme Medium: https://medium.com/the-language-of-vr/in-the-blink-of-a-mindprologue-7864c0474a29, 13 janvier 2016 ; https://medium.com/the-language-of-vr/in-the-blink- 
of-a-mind-attention-1fdff60fa045, 05 février 2016, consultation le 20 mai 2019. Scott Macaulay, « Cut: Jessica Brillhart on Editing VR» dans Fimmaker, 28 octobre 2015, https:// filmmakermagazine.com/96090-look-into-the-cut/\#.XOIyKFP7TOQ, consultation le 20 mai 2019. Benjamin Hoguet et Philipe Fuchs ont également mentionné Probabilistic Experiential Editing, voir Hoguet, op. cit, p. 134-147; Fuchs, Théorie de la réalité virtuelle -Les véritables usages, op. cit. p. 333-334.

34. André Bazin, op. cit., p. 152.

35. Jacques Araszkiwiez, «La Génèse de la théâtralité » dans Cinéma et théâtralité, sous la direction de Christine Hamon-Sirejols, Jacques Gerstenkorn, André Gardies, Lyon : Aléas, 1994, p. 22.

36. Pierre Lévy, op. cit., p. 31.

37. Buff, «The Deserted. Tsai Ming-liang: Je peux vous laisser voir plus, plus attentivement et plus librement» (Jiazailanrsi Tsai Ming-liang: yuanlai wokeyi rangni kan de genduo, genzixi, genziyou 《家在蘭若寺》蔡明亮：員來我可以讓你看得更多、更仔細、更自由), publié sur le site Hypesphere: https://www.hypesphere.com/news/14957, 31 janvier 2019, consultation le 31 janvier 2020.

\section{ABSTRACTS}

Abstract

360 cinema has been developing since 2016 in Taiwan. Tsai Ming-Liang has initiated a narrative experiment with this "new" technology and filmmakers are also joining him in this phase of exploration. When innovation and breakthrough are are highlighted to distinguish 360 virtual reality films from so-called conventional cinema, they are not considered as cinema for some filmmaker, as Alejandro González Iñárritu: "Virtual reality, even when it's visual, is exactly all what cinema is not". However, are these two characteristics always supported in the sense of technological evolution as well as visual culture? Does 360 cinema immediately mark a technological breakthrough as well as visual culture with cinema? How can we still identify cinematographic legacies in a new regime of visual representation?

We find in the works The Deserted (2017) by Tsai Ming-Liang, Your Spiritual Temple Sucks (2017) by John Hsu, Afterimage for Tomorrow (2018) by Singing Chen, M. Buddha (2018) by Chung Lee and Home (2019) by Kidning Hsu that a tension persists in the relationship between theater, cinema and 360 images. Does it prove this new "cinema" still remains in a state of prior institutionalization (in the sense of Gaudreault)? Or does it constitue an aesthetic specific to 360 cinema? An archaeological perspective will accompany the analyzes of these Taiwanese works, to reflect on the historicity of a cinema by dialoguing between its past and its future.

Résumé

Le cinéma $360^{\circ}$ se développe depuis 2016 à Taïwan. Tsai Ming-Liang a initié une expérimentation narrative avec cette "nouvelle" technologie et des cinéastes le rejoignent également dans cette phase d'exploration. Lorsque l'innovation et la rupture sont mises en avant pour distinguer les films en réalité virtuelle à $360^{\circ}$ du cinéma dit conventionnel, elles n'en font pas le cinéma pour certains cinéastes, comme Alejandro González Iñárritu: "Virtual reality, even when it's visual, is exactly all what cinema is not". Or, est-ce que ces deux caractéristiques sont toujours soutenue dans le sens de l'évolution technologique de même que la culture visuelle ? Comment peut-on 
encore identifier les héritages cinématographiques dans un nouveau régime de la représentation visuelle?

Nous trouvons dans les œuvres The Deserted (2017) de Tsai Ming-Liang, Your Spiritual Temple Sucks (2017) de John Hsu, Afterimage for Tomorrow (2018) de Singing Chen, Mr. Buddha (2018) de Chung Lee et Home (2019) de Kidning Hsu qu'une tension persiste dans la relation entre le théâtre, le cinéma et les images en 360. Prouve-t-elle que ce nouveau "cinéma" demeure encore dans un état d'avant institutionnalisation (au sens de Gaudreault) ? Ou constitue-t-elle une esthétique propre au cinéma 360 ? Un regard archéologique accompagnera les analyses de ces œuvres taïwanaises, pour penser une historicité d'un cinéma en dialoguant entre son passé et son devenir.

\section{AUTHOR}

\section{WEI-CHU SHIH}

Professeure assistante à l'université nationale centrale de Taiwan travaillant dans les domaines de l'esthétique du cinéma et l'éducation à l'image. L'auteure mène également le projet de cinéma $360^{\circ}$ intitule :The body in an offside position : the migration of the cinematographic language in $V R$ cinema. subventionné par le ministère de la science et de la technologie de Taïwan. 